\title{
8 BIT SINGLE CYCLE PROCESSOR
}

\author{
Praween K Sinha, Suyash Ahluwalia and Deepanshu Gupta \\ Department of Electronics and Communication Engineering, \\ Maharaja Agrasen Institute of Technology, Delhi, India.
}

\begin{abstract}
Processors are an integral part of the computer and electronics industry. Every computational unit contains some sort of processing circuit, designed to perform multiple operations on a single device and can be categorized based on its speed, flexibility and adaptability. RISC, CISC, Harvard and Von Neumann are some of these philosophies, emphasizing on a generalized approach for the design. This paper describes the design and implementation of a custom 8-bit single cycle processor on Xilinx Vivado Design suite, supporting 16 instructions, 256 bytes of internal storage, an Arithmetic Logic Unit and control unit. The module functionalities, Instruction set design and Data flow of the processor have also been discussed for a subset of instructions along with the simulation results.
\end{abstract}

Key words: 8-bit Processor, Microprocessor, Single cycle.

Cite this Article: Praween K Sinha, Suyash Ahluwalia and Deepanshu Gupta, 8 Bit Single Cycle Processor, International Journal of Computer Engineering and Technology, 10(2), 2019, pp. 160-168.

http://iaeme.com/Home/issue/IJCET?Volume=10\&Issue=2

\section{INTRODUCTION}

Computer architecture and design are one of the most advancing fields of technology, and with an increasing use of processing devices on daily basis, the goal is to establish a balance between performance and power consumption. The present work elaborates the design and verification of an 8-bit single cycle processor. It's concise and simple architecture allows quick instruction execution, eliminating the need of microcode and reducing latency. The instructions have been designed for low complexity, hence facilitating the flow of operations. Separate memory and buses for program and data adds to the same. This paper discusses different modules of the processor along with its instruction set and datapaths, organized as follows. Section 2 briefly describes the architecture, followed by the Instruction Set and Control matrix design in section 3 and 4 respectively. Section 5 contains implementation of top level as well as individual modules in Xilinx VIVADO and results have been presented in section 6. 


\section{ARCHITECTURE}

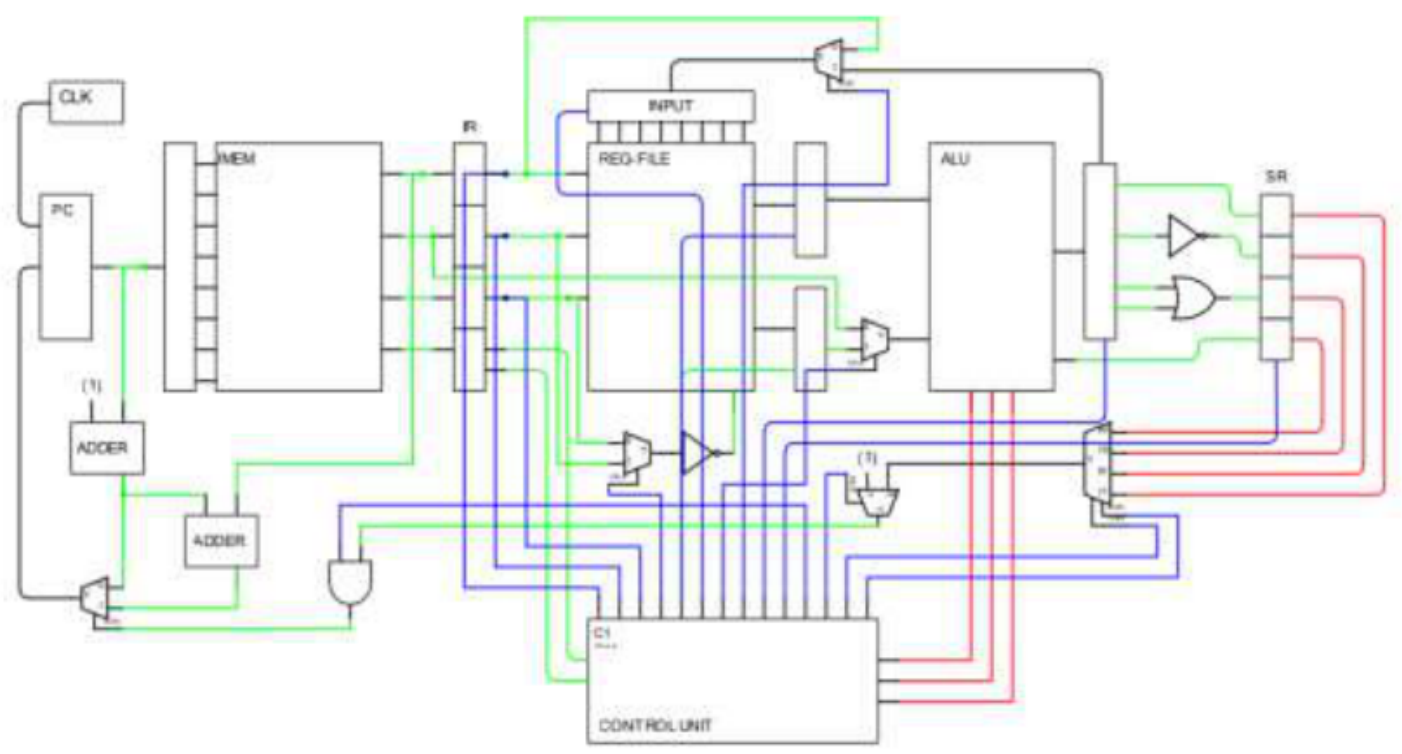

Figure 1 Processor architecture

The architecture of this 8-bit processor has been given in the Figure 1. Its main components include the Program Counter, followed by the Instruction Memory, the address decoders, the Register file, an 8-bit ALU and a Control Unit. All these components can be identified in the representation as well. The design incorporates separate program memory to store the instructions and register memory for storing data. All instructions are executed in one clock cycle and the 8-bit program counter maintains the address of every upcoming instruction from the stack of 256 memory locations. Once fetched, they are split, decoded and executed, following a distinguished datapath for each type. A major part of these instruction executions includes handling data in the form of variables, which is overseen by the ALU and Register file. The 8-bit Arithmetic and Logical Unit supports addition, subtraction, logical AND, OR and comparison operations for both, immediate and register type instructions, the results of which can be stored into internal registers. The Register file provides 256 bytes of internal storage along with the Load operation to directly write into memory. The input data for Arithmetic and Logical computations can be read in similar fashion, either from the Regfile or directly from the instruction register. Each instruction uses these described processor modules in a similar or different order, based on its type. The task of a Control Unit is to provide a viable datapath for the execution of these instructions. It reads 8 input signals from the Instruction Register and generates 14 output control bits that triggers the data path required for the execution of every instruction.

\section{INSTRUCTION SET DESIGN}

The processor has been designed to support a set of 16 instructions, each of which has a size of 32 bits, which will be used to identify the operations that are to be performed and the memory locations that need to be accessed in order to execute those operations. The set includes 3 types of instructions- Register type, immediate type and branch type along with a load instruction that writes the specified value directly into Data memory. Table 1 contains the format for each type of instruction. The Instruction Memory has 256 accessible memory locations, each with 4-byte word size. The higher 8 bits store the Control Unit opcode while the lower 24 bits are occupied by the addresses or data values. 
Table 1 Instruction formats

\begin{tabular}{|c|c|c|c|c|}
\hline Inst. Type & IR [31:24] & IR [23:16] (R1) & IR [15:8] (R2) & IR [7:0] (R3) \\
\hline Register type & Opcode & Read address 1 & Read address 2 & Write address \\
\hline Immediate type & Opcode & Read address 1 & Data & Write address \\
\hline Branch type & Opcode & Branch value & - & - \\
\hline Load & Opcode & Data & Write address & - \\
\hline
\end{tabular}

Each instruction is completed in the following steps- fetch, decode and execute. Instructions to be read are addressed using the program counter, fetched into the Instruction Register and split into 4 parts- the Opcode, Address/Data 1, Address/Data 2 and Write address. The opcode determines the operation and the multiplexed address bus carries the remaining address/data bits to Register memory for read and write operations. Whereas the branch instruction requires only the opcode and the branch value that leaves the lower 16 bits in don't care.

Table 2 Instruction Set design

\begin{tabular}{|c|c|c|c|c|c|}
\hline $\begin{array}{l}\text { Inst. } \\
\text { No }\end{array}$ & IR1[31:24] & IR2[23:16] & IR3[15:8] & IR4[7:0] & OPERATION \\
\hline 1 & Add & R1 & $\mathrm{R} 2$ & $\mathrm{R} 3$ & $\mathrm{R} 3=\mathrm{R} 1+\mathrm{R} 2$ \\
\hline 2 & Sub & $\mathrm{R} 1$ & $\mathrm{R} 2$ & R3 & $\mathrm{R} 3=\mathrm{R} 1-\mathrm{R} 2$ \\
\hline 3 & And & $\mathrm{R} 1$ & $\mathrm{R} 2$ & R3 & $\mathrm{R} 3=\mathrm{R} 1 \& \mathrm{R} 2$ \\
\hline 4 & Or & $\mathrm{R} 1$ & $\mathrm{R} 2$ & R3 & $\mathrm{R} 3=\mathrm{R} 1 \mid \mathrm{R} 2$ \\
\hline 5 & Addi & $\mathrm{R} 1$ & $X$ & R3 & $\mathrm{R} 3=\mathrm{X}+\mathrm{R} 2$ \\
\hline 6 & Subi & R1 & $\mathrm{X}$ & $\mathrm{R} 3$ & $\mathrm{R} 3=\mathrm{X}-\mathrm{R} 2$ \\
\hline 7 & Andi & R1 & $\mathrm{X}$ & $\mathrm{R} 3$ & $\mathrm{R} 3=\mathrm{X} \& \mathrm{R} 2$ \\
\hline 8 & Ori & $\mathrm{R} 1$ & $X$ & $\mathrm{R} 3$ & $\mathrm{R} 3=\mathrm{X} \mid \mathrm{R} 2$ \\
\hline 9 & SLT & R1 & $\mathrm{R} 2$ & R3 & $\begin{array}{c}\mathrm{R} 3=1 \quad \text { if } \quad \mathrm{R} 1<\mathrm{R} 2 \quad \text { else } \\
\mathrm{R} 3=0\end{array}$ \\
\hline 10 & SLTi & R1 & $\mathrm{X}$ & R3 & $\begin{array}{ccc}\mathrm{R} 3=1 \quad \text { if } \quad \mathrm{R} 1<\mathrm{X} & \text { else } \\
\mathrm{R} 3=0 & \\
\end{array}$ \\
\hline 11 & Load & $\mathrm{X}$ & $\mathrm{R} 2$ & - & Store $\mathrm{X}$ in address of $\mathrm{R} 2$ \\
\hline 12 & BRZ & $X$ & - & - & $\begin{array}{c}\mathrm{PC}=\mathrm{PC}+\mathrm{X}+1 \text { if Result is } \\
\text { Zero }\end{array}$ \\
\hline 13 & BRN & $\mathrm{X}$ & - & - & $\begin{array}{r}\mathrm{PC}=\mathrm{PC}+\mathrm{X}+1 \text { if Result is } \\
\text { Negative }\end{array}$ \\
\hline 14 & BRP & $\mathrm{X}$ & - & - & $\begin{array}{r}\mathrm{PC}=\mathrm{PC}+\mathrm{X}+1 \text { if } \text { Result is } \\
\text { Positive }\end{array}$ \\
\hline 15 & $\mathrm{BRO}$ & $X$ & - & - & $\begin{array}{c}\mathrm{PC}=\mathrm{PC}+\mathrm{X}+1 \text { if Result is } \\
\text { Overflowed }\end{array}$ \\
\hline 16 & BRU & $\mathrm{X}$ & - & - & $P C=P C+X+1 \quad$ in any case \\
\hline
\end{tabular}

The ALU supports 5 primary operations of addition, subtraction, logical AND, logical OR and 'Set on Less Than' (SLT). All these operations are supported in both, register and immediate type instructions. Table 2 contains set of all 16 instructions along with the operations they perform and Table 3 lists different opcodes combinations for all these instructions. The Branch instruction controls the data flow in two ways - conditionally or unconditionally. Unconditional branching increases the program counter by the value given in instruction, whereas conditional branching verifies the set condition with the contents of Status Register. Conditional branching involves 4 defined flags stored in Status Register - Negative, Positive, 
Zero and Overflow. The program flow will branch by the given value only if the required condition agrees with the SR contents, else PC will only be incremented by 1 .

For example, addition can be performed using the instruction 40000104h. The opcode for addition, as given in Table 3 is $40 \mathrm{~h}$, further followed by address location $00 \mathrm{~h}, 01 \mathrm{~h}$ and $04 \mathrm{~h}$. Therefore, contents of $00 \mathrm{~h}$ and $01 \mathrm{~h}$ memory locations will be added using the ALU and the final result will be stored in $04 \mathrm{~h}$. In another instance, the instruction 050900xxh will load 09h in zeroth location of memory. The first 8 bits $(05 \mathrm{~h})$ provide the opcode for Load instruction, followed by the data value and write address.

Table 3 Instruction opcodes (IR [31:24])

\begin{tabular}{|c|c|c|c|c|c|c|c|c|c|}
\hline Inst no. & Instruction & Op2 & Op1 & Op0 & Ctr14 & Ctr13 & Ctr12 & Ctr11 & Ctr10 \\
\hline 1 & Add & 0 & 1 & 0 & 0 & 0 & 0 & 0 & 0 \\
\hline 2 & Sub & 1 & 1 & 0 & 0 & 0 & 0 & 0 & 0 \\
\hline 3 & And & 0 & 0 & 0 & 0 & 0 & 0 & 0 & 0 \\
\hline 4 & Or & 0 & 0 & 1 & 0 & 0 & 0 & 0 & 0 \\
\hline 5 & Addi & 0 & 1 & 0 & 0 & 0 & 1 & 0 & 0 \\
\hline 6 & Subi & 1 & 1 & 0 & 0 & 0 & 1 & 0 & 0 \\
\hline 7 & Andi & 0 & 0 & 0 & 0 & 0 & 1 & 0 & 0 \\
\hline 8 & Ori & 0 & 0 & 1 & 0 & 0 & 1 & 0 & 0 \\
\hline 9 & SLT & 1 & 1 & 1 & 0 & 0 & 0 & 0 & 0 \\
\hline 10 & SLTi & 1 & 1 & 1 & 0 & 0 & 1 & 0 & 0 \\
\hline 11 & Load & 0 & 0 & 0 & 0 & 0 & 1 & 0 & 1 \\
\hline 12 & BRZ & 0 & 0 & 0 & 0 & 0 & 1 & 1 & 0 \\
\hline 13 & BRN & 0 & 0 & 0 & 0 & 1 & 1 & 1 & 0 \\
\hline 14 & BRP & 0 & 0 & 0 & 1 & 0 & 1 & 1 & 0 \\
\hline 15 & BRO & 0 & 0 & 0 & 1 & 1 & 1 & 1 & 0 \\
\hline 16 & BRU & 0 & 0 & 0 & 0 & 0 & 1 & 1 & 1 \\
\hline
\end{tabular}

\section{DATAPATH AND CONTROL MATRIX}

The datapath is a collection of processor elements that allow the transfer of data and addresses via buses and functioning of operating units like ALU by triggering the control elements in its path such as multiplexers, decoders etc. In the architecture given in figure 1, the 14 control bits generated by the control unit coordinate these buses and elements. The input $\mathrm{c}$ [7:0] of the control unit are the higher bits of Instruction Register (IR [31:24] and can be split into 2 segments, 3 bits and 5 bits each. The higher 3 bits (c [7:5]) consist of the ALU opcode whereas the lower 5 bits (c [4:0]) are required to compute the control unit outputs (C [1:14]).

Table 4 Control bits and their functions

\begin{tabular}{|c|c|c|}
\hline $\begin{array}{c}\text { Output } \\
\text { Bit }\end{array}$ & Equivalent logic & Bit Function \\
\hline $\mathrm{C}[1]$ & $(\sim \mathrm{c}[1]) \&(\sim \mathrm{c}[0])$ & Address decoder enable for R1 register of instruction \\
\hline $\mathrm{C}[2]$ & $(\sim \mathrm{c}[2]) \mid((\sim \mathrm{c}[1]) \& \mathrm{c}[0])$ & Address decoder enable for $\mathrm{R} 2$ register of instruction \\
\hline $\mathrm{C}[3]$ & $(\sim \mathrm{c}[1]) \&(\sim \mathrm{c}[0])$ & Address decoder enable for $\mathrm{R} 3$ register of instruction \\
\hline $\mathrm{C}[4]$ & $(\sim \mathrm{c}[0]) \mid \mathrm{c}[1]$ & Mux select for choosing $\mathrm{R} 2 / \mathrm{R} 3$ register $(0=\mathrm{R} 2,1=\mathrm{R} 3)$ \\
\hline
\end{tabular}


8 Bit Single Cycle Processor

\begin{tabular}{|c|c|c|}
\hline $\mathrm{C}[5]$ & $(\sim \mathrm{c}[1]) \&(\sim \mathrm{c}[0])$ & Regfile output enable for read operation \\
\hline $\mathrm{C}[6]$ & $(\sim \mathrm{c}[1])$ & Regfile input enable for write operation \\
\hline $\mathrm{C}[7]$ & $(\sim \mathrm{c}[2]) \mid(\mathrm{c}[1] \mid \mathrm{c}[0])$ & Mux select for Regfile/IR read $(0=$ IR, $1=$ Regfile out $)$ \\
\hline $\mathrm{C}[8]$ & $(\sim \mathrm{c}[1]) \& \mathrm{c}[0]$ & Mux select for Regfile input $(0=\mathrm{ALU}, 1=\mathrm{R} 1)$ \\
\hline $\mathrm{C}[9]$ & $(\sim \mathrm{c}[1]) \&(\sim \mathrm{c}[0])$ & ALU output $(\mathrm{A}[7: 0])$ enable \\
\hline $\mathrm{C}[10]$ & $(\sim \mathrm{c}[0]) \mid \mathrm{c}[1]$ & Status register enable \\
\hline $\mathrm{C}[11]$ & $\mathrm{c}[1]$ & Branch instruction enable $(1=$ branch type instruction) \\
\hline $\mathrm{C}[12]$ & $\mathrm{c}[1] \& \mathrm{c}[0]$ & Branch type select $(0=$ Conditional, $1=$ Unconditional $)$ \\
\hline $\mathrm{C}[13]$ & $\mathrm{c}[3]$ & Mux select 1 for branch condition selection \\
\hline $\mathrm{C}[14]$ & $\mathrm{c}[4]$ & Mux select 2 for branch condition selection \\
\hline
\end{tabular}

Table 4 consist the equivalent logic of control bits along with their functional description whereas Table 5 contains complete control matrix for the instruction set.

The general datapath for any execution follows a routine which includes reading PC, fetching instruction from Imem into IR, decoding the IR into R1, R2, R3 and c[7:0]. These steps are followed by read operations in Regfile, ALU processing and write operations into memory in the same chronological order. For instructions such as load or branch, aforementioned operations can be skipped as they do not require any arithmetic or logical computing, yet alone read any data from the Regfile. Once completed, the instruction ends with incrementing the contents of program counter by a value deemed necessary. Until the START bit is set high, the processor stays in OFF state, allowing us to write into program memory. Once activated, the instructions will be fetched and executed in the order specified by the program. This flow of execution relies entirely on Control Unit to maneuver the datapath according to its requirements.

Table 5 Control matrix

\begin{tabular}{|c|c|c|c|c|c|c|c|c|c|c|c|c|c|c|}
\hline Instruction & C1 & C2 & C3 & C4 & C5 & C6 & C7 & C8 & C9 & C10 & C11 & C12 & C13 & C14 \\
\hline Add & 1 & 1 & 1 & 1 & 1 & 1 & 1 & 0 & 1 & 1 & 0 & 0 & 0 & 0 \\
\hline Sub & 1 & 1 & 1 & 1 & 1 & 1 & 1 & 0 & 1 & 1 & 0 & 0 & 0 & 0 \\
\hline And & 1 & 1 & 1 & 1 & 1 & 1 & 1 & 0 & 1 & 1 & 0 & 0 & 0 & 0 \\
\hline Or & 1 & 1 & 1 & 1 & 1 & 1 & 1 & 0 & 1 & 1 & 0 & 0 & 0 & 0 \\
\hline Addi & 1 & 0 & 1 & 1 & 1 & 1 & 0 & 0 & 1 & 1 & 0 & 0 & 0 & 0 \\
\hline Subi & 1 & 0 & 1 & 1 & 1 & 1 & 0 & 0 & 1 & 1 & 0 & 0 & 0 & 0 \\
\hline Andi & 1 & 0 & 1 & 1 & 1 & 1 & 0 & 0 & 1 & 1 & 0 & 0 & 0 & 0 \\
\hline Ori & 1 & 0 & 1 & 1 & 1 & 1 & 0 & 0 & 1 & 1 & 0 & 0 & 0 & 0 \\
\hline SLT & 1 & 1 & 1 & 1 & 1 & 1 & 1 & 0 & 1 & 1 & 0 & 0 & 0 & 0 \\
\hline SLTi & 1 & 0 & 1 & 1 & 1 & 1 & 0 & 0 & 1 & 1 & 0 & 0 & 0 & 0 \\
\hline Load & 0 & 1 & 0 & 0 & 0 & 1 & 1 & 1 & 0 & 0 & 0 & 0 & 0 & 0 \\
\hline BRZ & 0 & 0 & 0 & 1 & 0 & 0 & 1 & 0 & 0 & 1 & 1 & 0 & 0 & 0 \\
\hline
\end{tabular}




\begin{tabular}{|l|l|l|l|l|l|l|l|l|l|l|l|l|l|l|}
\hline BRN & 0 & 0 & 0 & 1 & 0 & 0 & 1 & 0 & 0 & 1 & 1 & 0 & 1 & 0 \\
\hline BRP & 0 & 0 & 0 & 1 & 0 & 0 & 1 & 0 & 0 & 1 & 1 & 0 & 0 & 1 \\
\hline BRO & 0 & 0 & 0 & 1 & 0 & 0 & 1 & 0 & 0 & 1 & 1 & 0 & 1 & 1 \\
\hline BRU & 0 & 0 & 0 & 1 & 0 & 0 & 1 & 0 & 0 & 1 & 1 & 1 & 0 & 0 \\
\hline
\end{tabular}

For instance, in a register type addition instruction (Add), the first step will be fetching the 32 -bit instruction from Imem into the IR. Its unique opcode $(40 \mathrm{~h})$ will generate 14 control bits and address bus will access the remaining 24 bits for address decoding, enabled by the $\mathrm{C}[1]$, $\mathrm{C}[2]$ and $\mathrm{C}[3]$ bits. $\mathrm{C}[4]$ will be high, selecting R3 as the write address and $\mathrm{C}[5], \mathrm{C}[6]$, will both be enabled to facilitate input and output operations on Regfile. Then, the ALU will receive its opcode and perform addition on data fetched from memory and store the results in A [7:0], along with the flags in SR. Since the instruction is a register type instruction, C[7] will remain high to fetch ALU input from memory. For an immediate type instruction, the second input of ALU will be extracted from R2 instead of Regfile. C [8], C[9] and C[10] are set to 0, 1 and 1 respectively to write the ALU output back into Regfile and set the SR flags. Remaining 4 bits are enabled only in case of branch type instructions and hence, will be low here. Once the results are stored and SR configured, the PC increments itself by 1 to fetch the address of next instruction from Imem. Compared to Register type, the immediate type instructions require two major changes, in $\mathrm{C}[2\}$ and $\mathrm{C}[7]$ bits, setting them both to low. This will ensure R2 to be treated as a data input for ALU instead of a decoded address for Regfile.

\section{IMPLEMENTATION AND RESULTS}

The main logic modules consisting independent functioning capabilities were designed and verified rigorously using Xilinx VIVADO Design Suite. The working and simulations of these modules have been discussed here.

\subsection{Arithmetic \& Logic Unit}

As the name suggests, ALU performs calculations and logical operations on 8-bit signed values. Its design can be segregated into 1-bit ALU cells that supports 5 primary operations- Add, Sub, AND, OR and SLT. The execution of these operations is controlled by the 3-bit ALU opcode (same as IR[31:29] for all instructions that require ALU), as shown in figure 2.
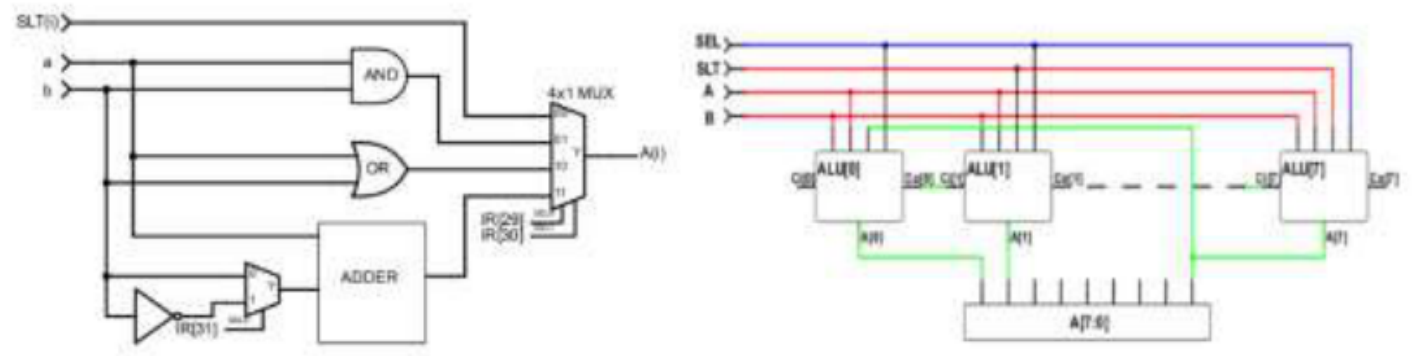

Figure 2 Arithmetic \& Logic Unit

The SLT operation, that results in output value 1 when the left operand is smaller than right one ( $\mathrm{a}$ SLT $\mathrm{b}=1$, if $\mathrm{a}<\mathrm{b}$, else 0 ), performs subtraction and stores the MSB of output into A[0] while setting all other bits to zero. Therefore, only when $a-b<0$, the negative answer will result in a high MSB that will be low otherwise. Figure 2 also shows the arrangement of individual cells to design the ALU. Once the result is computed and stored, the Status Register is configured accordingly. The 4-bit SR has the following flags- Negative, Positive, Zero and 
Overflow (N, P, Z, O). SR retains its previous value in case of low ALU enable or incorrect opcode and the same can be verified from figure 3, given below.

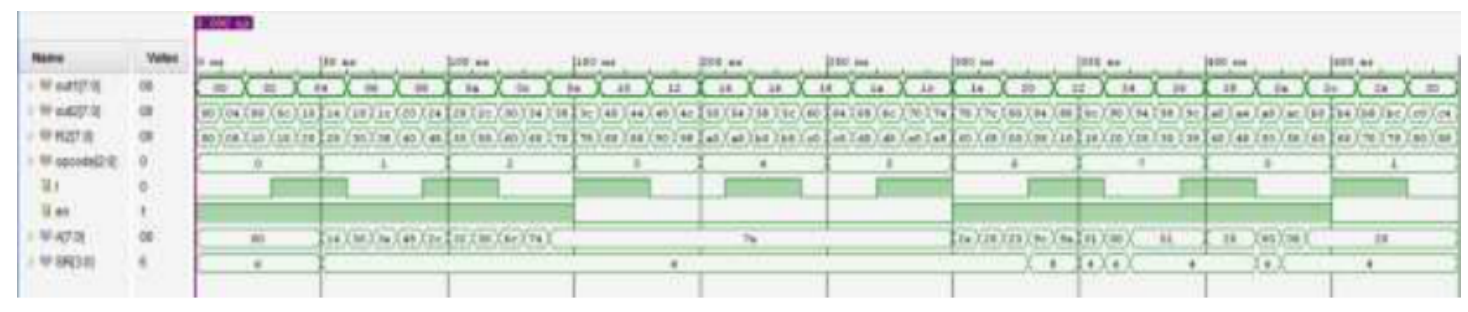

Figure 3 ALU simulation

\subsection{Register Memory}

The Register Memory has 256 bytes of storage available, stacked in the arrangement shown in figure 4, along with its standard SRAM cell diagram. Each cell requires 3 inputs- Data, rw (1= read, $0=$ write) and enable. The Write and Read operations cannot occur simultaneously that allows us to control rw bits multiplexed with the address decoder for R3, without needing any extra signals ( $\mathrm{rw}=\sim \mathrm{C}[4])$.

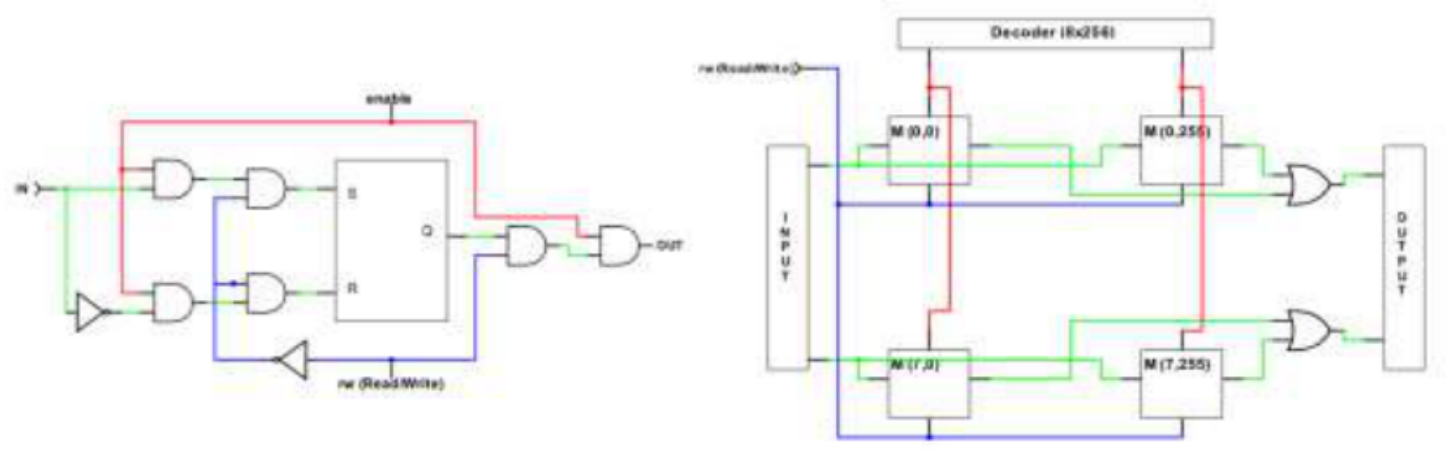

Figure 4 Register memory structure

Figure 5 contains the results of memory simulations for different scenarios involving different control bits configurations and memory locations which have an affect on the execution of Read/Write operations.

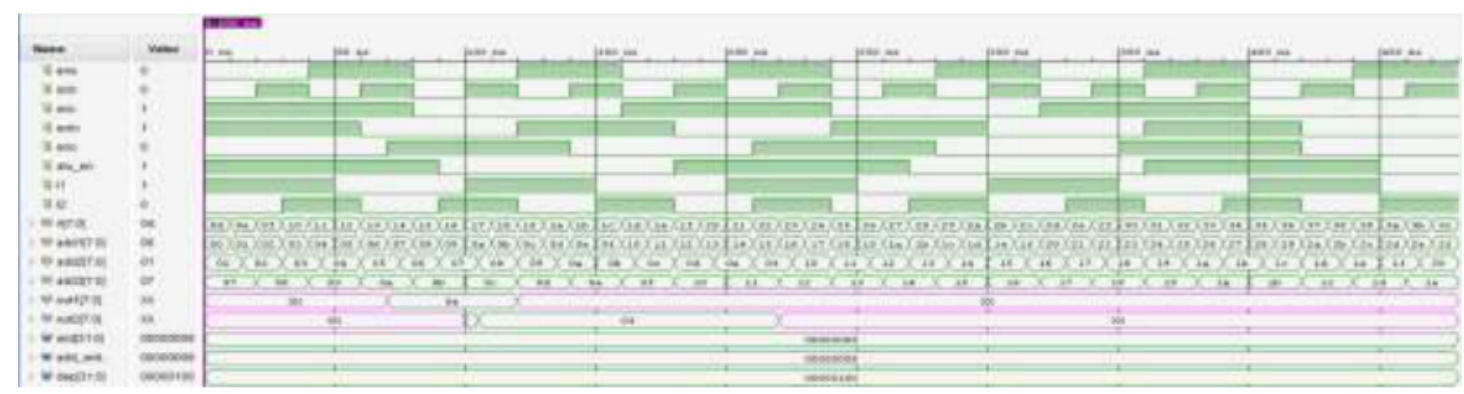

Figure 5 Memory simulation

\subsection{Instruction Memory \& Program Counter}

Based on Harvard Architecture, the design incorporates separate memory systems for data and program. The standard SRAM cell of IMEM resembles the one used for Data memory (see figure 4) and loading the program requires the rw bit to be set low, that is accomplished while $\mathrm{START}=0$. Once the program is loaded, the PC initiates with $00 \mathrm{~h}$ as soon as START is enabled. 
With each clock cycle, $\mathrm{PC}$ increases to $\mathrm{PC}+1$ or $\mathrm{PC}+(\mathrm{X}+1)$ for register and branch type instructions respectively. Figure 6 shows the simulation results for $\mathrm{PC}$ and figure 7 contains the simulations of IMEM.

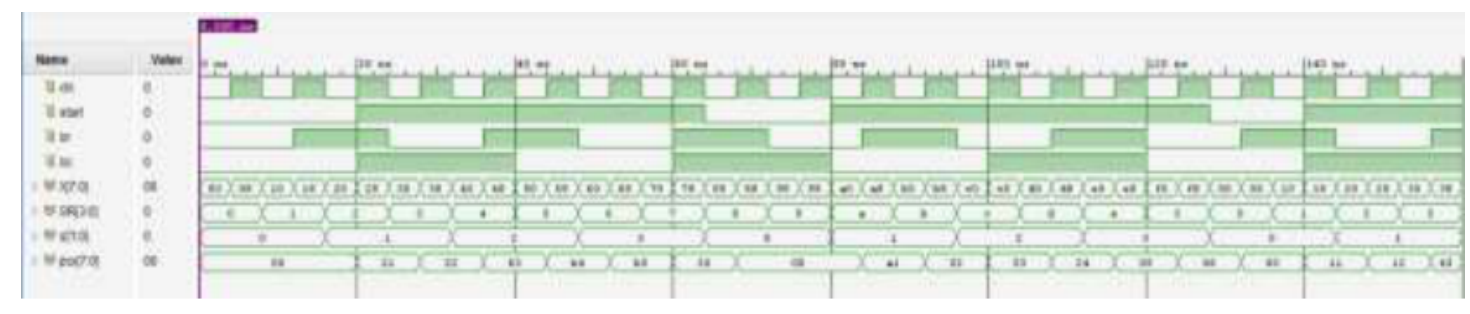

Figure 6 8-bit PC simulation

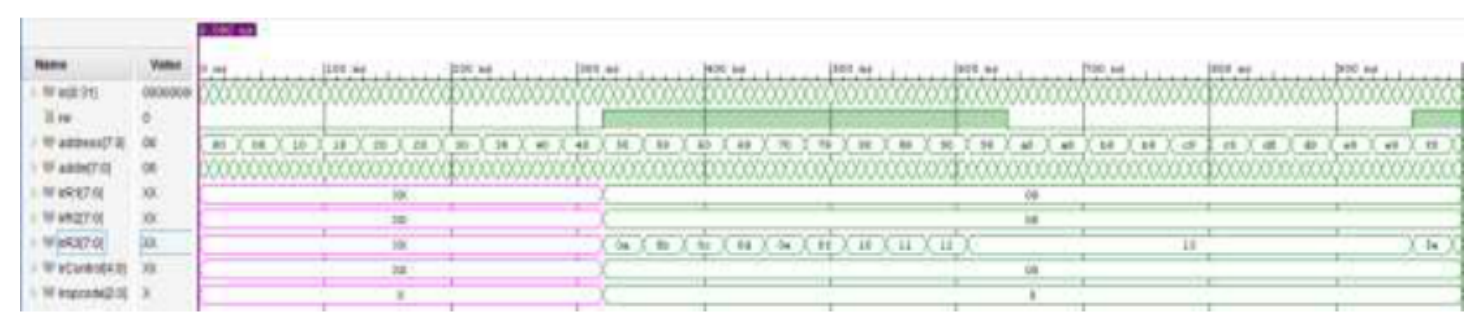

Figure 7 IMEM simulation

\subsection{Processor Design}

Simulation results for a series of instructions have been shown in figure 8. While IMEM_RW $=0(1=$ read, $0=$ write $)$, IMEM[INST_ADD] $=$ IMEM_IN. At START $=1$, PC initiates with $00 \mathrm{~h}$ and begins the execution. The testbench stores $09 \mathrm{~h}, 12 \mathrm{~h}, 1 \mathrm{Bh}$ and $24 \mathrm{~h}$ at $00,01,02$ and 03 respectively followed by repeated additions, results of which are seen in A[7:0] and flags are set in SR.

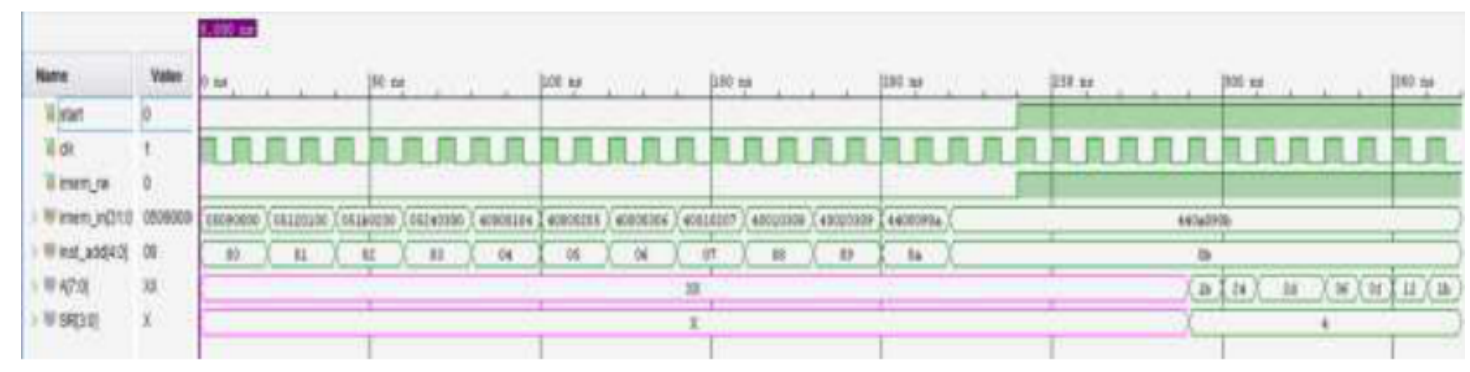

Figure 8 Top-level Processor simulation

\section{INSPIRATION AND COMPARISON}

The 8-bit Processor has been organized to improve simplicity while maintaining the necessary modules. While our design captures the same essence of architecture by Rane, Naik, Mehta (2017) using PC, Imem, Decoder, Regfile, ALU and CU as its key elements [1], it is worth noting that 8 internal registers and data memory have been replaced by 256 bytes of storage, serving both functionalities and hence eliminates the need for Load/Store instructions as both operations can be performed directly. This leads to an increase in Imem word size which incorporates 32 bits instead of 16. On the other hand, Instruction set has been modified to remove lw, sw and include additional immediate functions such as Andi \& Ori, along with SLT and conditional branching operations while keeping the opcode size equal to 3 bits. The ALU and Memory organization is inferred from Ayeh's design (2008) [2]. Though its key subtraction operations follow similar 2's compliment methods, the size has been increased to 8 bits to match the current processor architecture. According to Ayeh (2008), "As in a simple ROM, a decoder 
on the address lines selects a specific row of SRAM to be accessed at any time. Once the row is decided, 8 bits data is written in the 8 SRAM or road 8-bit data to next component.". An $8 \times 256$ decoder performs address selection instead of a $4 \times 16$ decoder, in a similar fashion. The increased decoder size compensates for more memory locations in the current design. This custom architecture, therefore provides more instructions and memory at your disposal while allowing the tradeoff for longer instructions and control unit output.

\section{CONCLUSION}

An 8-bit single cycle processor with 16 instructions set has been designed using Verilog HDL and verified using Xilinx VIVADO. All instructions are executed in one clock cycle. Doing so makes the design smaller, faster and easily understandable. All computations are done by 8-bit ALU, synchronization is done with an external clock signal, and all transitions occur at its positive edge, triggered by START and IMEM_RW bits. The simplicity, reduced latency and easy comprehensibility of the design can be utilized for educational purposes to understand the basic organization of processors, in hardware systems, mini sensor systems etc.

\section{REFERENCES}

[1] Rane, Naik, Mehta. 8 Bit Custom MIPS Microprocessor. International Journal of Computer Engineering \& Technology, 8(5), Sep-Oct 2017, pp. 23-30.

[2] Ayeh, Agbedanu, Morita, Adamo, Guturu. FPGA Implementation of an 8-bit Simple Processor. 2008 IEEE Region 5 Conference. April, 2008, pp. 1-5.

[3] Uma. Design and Performance Analysis of 8-bit RISC Processor using Xilinx Tool. International Journal of Engineering Research and Applications (IJERA), 2(2), Mar-Apr 2012, pp. 053-058.

[4] Gaonkar, M. Design of 16-Bit RISC Processor. International Journal of Scientific Research in Physics and Applied Sciences, 1(1), Feb 2017, pp. 25-30.

[5] Kaur, Anuj. 8 Bit RISC Processor Using Verilog HDL. Int. Journal of Engineering Research and Applications, 4(3), March 2014, pp. 417-422.

[6] Mano, Ciletti. Digital Design with an Introduction to the Verilog HDL, $5^{\text {th }}$ Edition, Pearson Education, 2013.

[7] Palnitkar. Verilog HDL A Guide to Digital Design and Synthesis, 2 ${ }^{\text {nd }}$ Edition, Sun Microsystems, 2003.

[8] Mano. Digital Logic and Computer Design, Pearson Education, 1979. 